\title{
Novel smacoviruses identified in the faeces of two wild felids: North American bobcat and African lion
}

\author{
Simona Kraberger ${ }^{1}$ (1) $\cdot$ Laurel Serieys $^{2,3,6} \cdot$ Nicolas Fountain-Jones $^{4} \cdot$ Craig Packer $^{5} \cdot$ Seth Riley ${ }^{6} \cdot$ Arvind Varsani $^{1,7,8,9}$
}

Received: 3 May 2019 / Accepted: 29 May 2019 / Published online: 22 June 2019

(c) Springer-Verlag GmbH Austria, part of Springer Nature 2019

\begin{abstract}
Smacoviruses are small circular single-stranded DNA viruses that appear to be prevalent in faeces of a range of animals and have also been found in a few insect species. In this study, we report the first viral genomes from faeces of free-roaming wild felids on two continents. Two smacoviruses were recovered from the faeces of two North American bobcats (Lynx rufus), and one was recovered from an African lion (Panthera leo). All three genomes are genetically different, sharing 59-69\% genome-wide sequence identity to other smacoviruses. These are the first full smacovirus genome sequences associated with a large top-end feline predator, and their presence in these samples suggests that feline faeces are a natural niche for the organisms that these viruses infect.
\end{abstract}

The Smacoviridae are a family of circular single-stranded DNA viruses that have been identified in the faecal excrement of many mammals and birds, and a few arthropods [19]. The genomes of smacoviruses range from 2.3 to $3 \mathrm{~kb}$ in size and contain at least two bidirectionally transcribed open reading frames (ORFs), which encode a replicationassociated protein (Rep) and a capsid protein (CP). These ORFs are separated by two intergenic regions, one of which contains an origin of replication. At present, more than 170 smacoviruses have been documented, with six established genera; Bovismacovirus, Cosmacovirus, Dragsmacovirus, Drosmacovirus, Huchismacovirus and Porprismacovirus. Recently, it was shown that DNA matching a smacovirus was found in the archaeon "Candidatus Methanomassiliicoccus intestinalis", suggesting that smacoviruses may infect faeces-dwelling archaea [7]. Despite the broad range

Handling Editor: William G. Dundon.

Accession numbers: MK796234-MK796236.

Electronic supplementary material The online version of this article (https://doi.org/10.1007/s00705-019-04329-3) contains supplementary material, which is available to authorized users.

Simona Kraberger

simona.kraberger@asu.edu

Arvind Varsani

arvind.varsani@asu.edu

Extended author information available on the last page of the article of animals with which smacoviruses have been associated, none have been documented in a member of the family Felidae. Felids, both domestic and wild, are known to harbour a multitude of viruses, including; feline immunodeficiency virus $[13,20]$, feline foamy virus $[4,11]$, feline leukaemia virus $[5,16]$, feline anellovirus $[9,21]$, feline gammaherpesvirus [2, 18], feline coronavirus [15], and several more.

In two separate studies, faecal samples were collected from bobcats (Lynx rufus) in California, USA, and African lions (Panthera leo) in the Serengeti National Park in Tanzania. Faecal samples described in this study were collected from three wild cats. One bobcat sample was collected during necropsy, the other from a live trap on the day of capture, and the African lion sample was collected shortly after defecation was observed. Viral DNA was extracted, and circular molecules were enriched from the faecal samples according to a previously described protocol [12]. Enriched DNA was sequenced on an Illumina 2500 platform, and contigs were generated through de novo assembly of paired-end reads using metaSPAdes v. 3.12.0 [1]. Three contigs with similarities to smacoviruses were identified in BLASTx searches and amplified from the three individual samples using abutting primers (Table 1) by PCR. The amplicons were then cloned and sequenced using the Sanger method [12].

We have tentatively named the three novel smacoviruses reported in this study "Lynx rufus associated smacovirus 1" (LruSmV 1, MK796234), "Lynx rufus associated smacovirus 2" (LruSmV 2, MK796235) and "Panthera leo associated smacovirus" (PISmV, MK796236). Their genomes are 2,435 
Table 1 Sample information and primers used for recovery of full genomes by PCR

\begin{tabular}{lllll}
\hline Sample source & Isolate name & Genome size $(\mathrm{nt})$ & Forward primer & Reverse primer \\
\hline Bobcat & Lynx rufus smacovirus 1 & 2,435 & TTTCAGCAAGCTAGTAAGAAG & TGTATTCATCCTTCCATTACCTTC \\
& & & GTGTTCATCGATGG & TCTATATCCGC \\
Bobcat & Lynx rufus smacovirus 2 & 2,595 & CAACTTACTGGTGATTCGTCCTCT & AGATACATTCTCATACACACCCTG \\
& & & AATTATCCG & ACCATAATACG \\
African lion & Panthera leo smacovirus & 2,650 & GTCCTTCTGTAGTATGCCATTTTA & CTATAGAAGGTCAAGGTACAGTTA \\
& & & TACACC & CAGAAG \\
\hline
\end{tabular}

nt, $2595 \mathrm{nt}$ and 2,650 nt, respectively, in size (Table 1). All contain two large open reading frames oriented in opposite directions, encoding the Rep and CP proteins. 174 complete smacovirus genome sequences available in the GenBank database were compiled and used for comparison with the three sequences reported here. Full-genome, Rep and CP amino acid sequence datasets were assembled and used to determine pairwise identity values using SDT 1.2 [14]. Genome-wide pairwise comparison showed that the three genomes share 54-57\% nucleotide sequence identity. With all other smacoviruses, the closest relative for each is as follows: LruSmV 1 shares 59\% sequence identity with chimpanzee associated porprismacovirus 2 (GQ351273) [3], LruSmV 2 shares $61 \%$ sequence identity with Chlorocebus cynosuros associated smacovirus (LC386199), and PISmV shares $67 \%$ sequence identity with sheep faeces associated smacovirus 3 (KT862219) [17] (Supplementary Data 1). Rep and CP amino acid comparison with all smacoviruses showed they share 39-63\% sequence identity in Rep and 35-60\% sequence identity in CP (Fig. 1 and Supplementary data 1).

Smacovirus Rep amino acid sequences were aligned using MAFFT [10], and the resulting alignment was used to construct a maximum-likelihood phylogenetic tree using PhyML 3.0 [8] with the best-fit amino acid model $\mathrm{rtREV}+\mathrm{G}+\mathrm{I}+\mathrm{F}$ selected using ProtTest [6]. Branches with less than 0.8 aLRT branch support were collapsed. The phylogenetic tree was rooted with the Rep sequences of the nanoviruses. Based on the taxonomic guidelines put forward by the Smacoviridae subcommittee for the International Committee on Taxonomy of Viruses [19], the results of phylogenetic analysis and sequence comparisons support the assignment of each of these three smacoviruses to a distinct species.
Fig. 1 Maximum-likelihood phylogenetic tree based on Rep protein sequences (left), displaying the six described genera. The LruSmV 1 Rep is most similar to that of Papio cynocephalus associated smacovirus (LC386204), sharing 38\% amino acid sequence identity. An expanded subtree of the Rep-based phylogeny of the porprismacoviruses is displayed on the right. Names in red letters indicate the feline-faeces-associated Reps of the genomes identified in this study. A pairwise amino acid sequence comparison of the two novel feline smacovirus Rep proteins in the genus Porprismacovirus to those of all other members of the genus is shown next to the phylogenetic tree

Phylogenetic analysis showed that the Rep of LruSmV 1 is divergent, forming a singleton clade that is closest to the huchismacoviruses. The Reps of LruSmV 2 and PISmV group in a clade consisting of the members of the genus Porprismacovirus (Fig. 1). The Rep proteins of LruSmV 2 and PISmV share a common ancestor with porcine associated porprismacovirus 10 (KT862225) and sheep faeces associated smacovirus 3 (KT862219), respectively (Fig. 1) [17]. It is worth noting that these two LruSmVs were recovered from the faeces of individual bobcats living in the Los Angeles area (California, USA), but they are genetically distinct from each other. If smacoviruses do in fact infect archaea [7], this may indicate that these two distinct viruses are hosted by different archaeal species. Here, we describe the first smacoviruses associated with felid faeces and show that they are highly diverse, representing three distinct species. Continued research in this area is needed to determine the true hosts of these viruses and to elucidate what relationship they have to other felid viruses and those infecting other animals. 

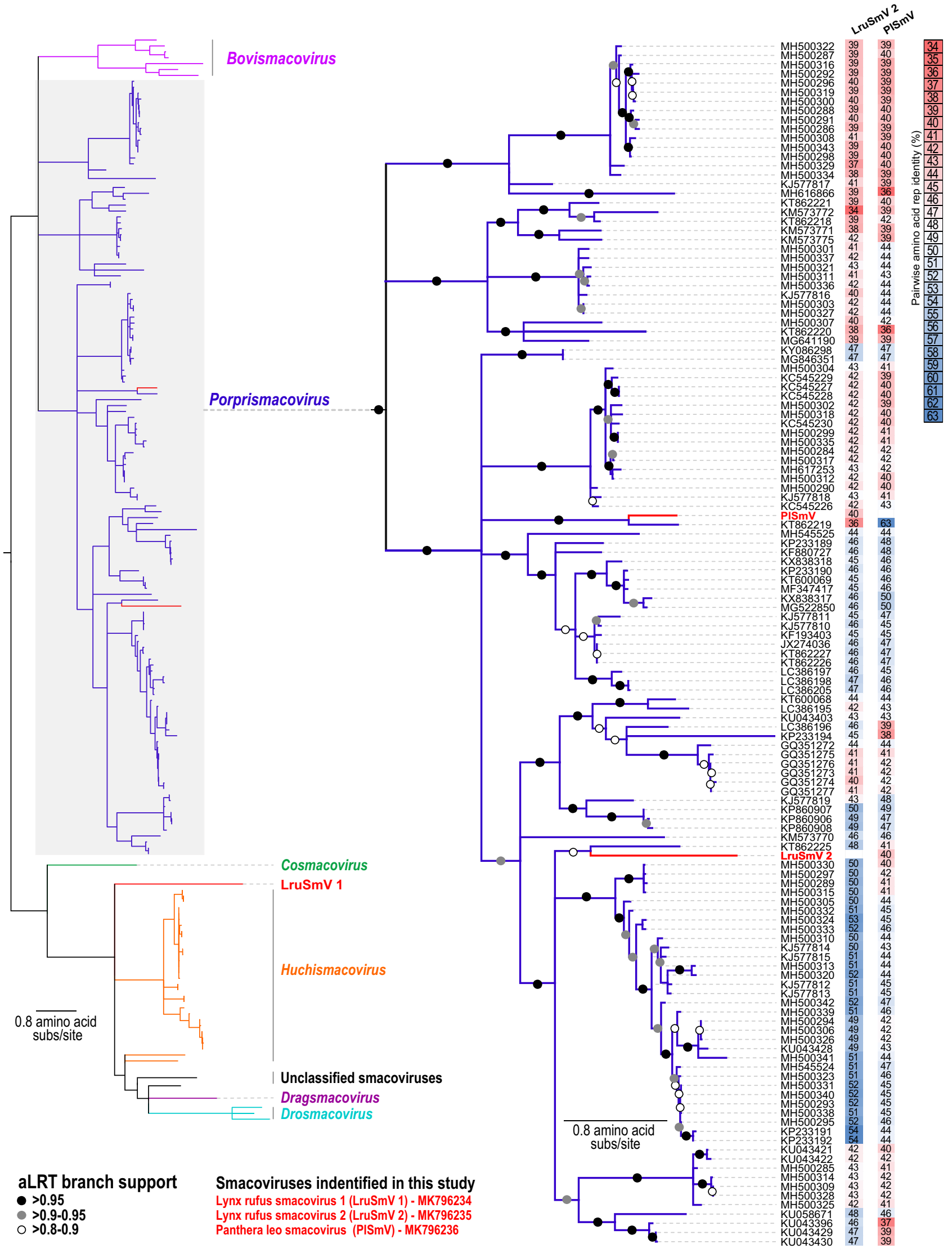
Acknowledgements Sample collection of bobcat faecal material was supported by the National Park Service and Santa Monica Mountains Fund, and Laurel Serieys was supported by a NSF graduate student fellowship.

\section{References}

1. Bankevich A, Nurk S, Antipov D, Gurevich AA, Dvorkin M, Kulikov AS, Lesin VM, Nikolenko SI, Pham S, Prjibelski AD (2012) SPAdes: a new genome assembly algorithm and its applications to single-cell sequencing. J Comput Biol 19:455-477

2. Beatty JA, Troyer RM, Carver S, Barrs VR, Espinasse F, Conradi O, Stutzman-Rodriguez $\mathrm{K}$, Chan $\mathrm{CC}$, Tasker S, Lappin MR (2014) Felis catus gammaherpesvirus 1; a widely endemic potential pathogen of domestic cats. Virology 460:100-107

3. Blinkova O, Victoria J, Li Y, Keele BF, Sanz C, Ndjango J-BN, Peeters M, Travis D, Lonsdorf EV, Wilson ML, Pusey AE, Hahn BH, Delwart EL (2010) Novel circular DNA viruses in stool samples of wild-living chimpanzees. J Gen Virol 91:74-86

4. Cavalcante L, Muniz C, Jia H, Augusto A, Troccoli F, Medeiros S, Dias C, Switzer W, Soares M, Santos A (2018) Clinical and molecular features of feline foamy virus and feline leukemia virus co-infection in naturally-infected cats. Viruses 10:702

5. Chiu ES, Kraberger S, Cunningham M, Cusack L, Roelke M, VandeWoude S (2019) Multiple introductions of domestic cat feline leukemia virus in endangered florida panthers. Emerg Infect Dis 25:92

6. Darriba D, Taboada GL, Doallo R, Posada D (2011) ProtTest 3: fast selection of best-fit models of protein evolution. Bioinformatics 27:1164-1165

7. Díez-Villaseñor C, Rodriguez-Valera F (2019) CRISPR analysis suggests that small circular single-stranded DNA smacoviruses infect Archaea instead of humans. Nat Commun 10:294

8. Guindon S, Dufayard J-F, Lefort V, Anisimova M, Hordijk W, Gascuel O (2010) New algorithms and methods to estimate maximum-likelihood phylogenies: assessing the performance of PhyML 3.0. Syst Biol 59:307-321

9. Jarošová V, Hrazdilová K, Filipejová Z, Schánilec P, Celer V (2015) Whole genome sequencing and phylogenetic analysis of feline anelloviruses. Infect Genet Evol 32:130-134

10. Katoh K, Misawa K, Ki Kuma, Miyata T (2002) MAFFT: a novel method for rapid multiple sequence alignment based on fast Fourier transform. Nucleic Acids Res 30:3059-3066

11. Kehl T, Bleiholder A, Roßmann F, Rupp S, Lei J, Lee J, Boyce W, Vickers W, Crooks K, VandeWoude S, Löchelt M
(2013) Complete genome sequences of two novel puma concolor foamy viruses from california. Genome Announcements 1:e00201-e00212

12. Kraberger S, Waits K, Ivan J, Newkirk E, VandeWoude S, Varsani A (2018) Identification of circular single-stranded DNA viruses in faecal samples of Canada lynx (Lynx canadensis), moose (Alces alces) and snowshoe hare (Lepus americanus) inhabiting the Colorado San Juan Mountains. Infect Genet Evol 64:1-8

13. Lee J, Malmberg JL, Wood BA, Hladky S, Troyer R, Roelke M, Cunningham M, McBride R, Vickers W, Boyce W, Boydston E, Serieys L, Riley S, Crooks K, VandeWoude S (2017) Feline immunodeficiency virus cross-species transmission: implications for emergence of new lentiviral infections. J Virol. https://doi. org/10.1128/JVI.02134-16

14. Muhire BM, Varsani A, Martin DP (2014) SDT: a virus classification tool based on pairwise sequence alignment and identity calculation. PLoS One 9:e108277

15. Myrrha LW, Silva FMF, Peternelli EFdO, Junior AS, Resende M, Almeida MRd (2011) The paradox of feline coronavirus pathogenesis: a review. Adv Virol. https://doi.org/10.1155/2011/10984 9

16. Powers JA, Chiu ES, Kraberger SJ, Roelke-Parker M, Lowery I, Erbeck K, Troyer R, Carver S, VandeWoude S (2018) Feline leukemia virus disease outcomes in a domestic cat breeding colony: relationship to endogenous FeLV and other chronic viral infections. J Virol. https://doi.org/10.1128/JVI.00649-18

17. Steel O, Kraberger S, Sikorski A, Young LM, Catchpole RJ, Stevens AJ, Ladley JJ, Coray DS, Stainton D, Dayaram A, Julian L, van Bysterveldt K, Varsani A (2016) Circular replication-associated protein encoding DNA viruses identified in the faecal matter of various animals in New Zealand. Infect Genet Evol 43:151-164

18. Troyer RM, Beatty JA, Stutzman-Rodriguez KR, Carver S, Lozano CC, Lee JS, Lappin MR, Riley SP, Serieys LE, Logan KA (2014) Novel gammaherpesviruses in North American domestic cats, bobcats and pumas: identification, prevalence and risk factors. J Virol 88(8):3914-3924

19. Varsani A, Krupovic M (2018) Smacoviridae: a new family of animal-associated single-stranded DNA viruses. Arch Virol 163(7):2005-2015

20. Winkler IG, Löchelt M, Flower RLP (1999) Epidemiology of feline foamy virus and feline immunodeficiency virus infections in domestic and feral cats: a seroepidemiological study. J Clin Microbiol 37:2848-2851

21. Zhang W, Wang H, Wang Y, Liu Z, Li J, Guo L, Yang S, Shen Q, Zhao X, Cui L, Hua X (2016) Identification and genomic characterization of a novel species of feline anellovirus. Virol J 13:146

Publisher's Note Springer Nature remains neutral with regard to jurisdictional claims in published maps and institutional affiliations.

\section{Affiliations}

\section{Simona Kraberger ${ }^{1}\left(10 \cdot\right.$ Laurel Serieys $^{2,3,6} \cdot$ Nicolas Fountain-Jones $^{4} \cdot$ Craig Packer $^{5} \cdot$ Seth Riley $^{6} \cdot$ Arvind Varsani $^{1,7,8,9}$}

\author{
Laurel Serieys \\ LaurelSerieys@gmail.com \\ Nicolas Fountain-Jones \\ nfj@umn.edu \\ Craig Packer \\ packer@umn.edu \\ Seth Riley \\ seth_riley@nps.gov
}

1 The Biodesign Center for Fundamental and Applied Microbiomics, Arizona State University, Tempe, AZ 85287, USA

2 Department of Biological Sciences, Institute for Communities and Wildlife in Africa, University of Cape Town, Rondebosch, Private Bag X3, Cape Town 7701, South Africa

3 Environmental Studies, University of California, Santa Cruz, Campus Mail Stop, 1153 High Street, Santa Cruz, CA, USA 
4 Department of Veterinary Population Medicine, University of Minnesota, 1365 Gortner Avenue, Saint Paul, MN 55108, USA

5 Department of Ecology Evolution and Behavior, University of Minnesota, Saint Paul, MN 55408, USA

6 Santa Monica Mountains National Recreation Area, National Park Service, Thousand Oaks, CA 91360, USA
7 School of Life Sciences, Arizona State University, Tempe, AZ 85287, USA

8 Center for Evolution and Medicine, Arizona State University, Tempe, AZ 85287, USA

9 Structural Biology Research Unit, Department of Clinical Laboratory Sciences, University of Cape Town,

Cape Town 7925, South Africa 